\title{
Malaria and HIV sero-positivity: study on selected individuals at a tertiary healthcare centre in Port Harcourt, Nigeria
}

\author{
Iheanyi O. Okonko, Anwuli U. Osadebe, Eberechukwu M. Okoli, Ego D. Eke \\ Department of Microbiology, University of Port Harcourt, Choba, Nigeria
}

\section{Summary}

Over $50 \%$ of the global population is vulnerable to malaria infection. An estimated 300 million malaria cases occur annually in the tropics with $90 \%$ of these in the sub-Sahara, a region that already suffers the greatest burden of HIV-1 infection. This study assessed the prevalence of HIV and malaria infections in a cohort of 200 undergraduates, consisting of 100 females and 100 males, attending the Health Centre at the University of Port Harcourt, Nigeria. Blood samples were screened for the presence of malaria and HIV, using SD Boline malaria Antigen P.F. test kit and Determine $^{\circledR}$ HIV one step rapid Kit. The overall prevalence for HIV in this study was $2.5 \%$ while the overall prevalence of malaria was $1.5 \%$. The prevalence of HIV based on gender was $2.0 \%$ and $3 \%$ for females and males, respectively. For malaria, these values were $2.0 \%$ and $1.0 \%$ for females and males, respectively. No incident of co-infection was recorded in this study. The findings did not indicate any relationship between HIV seropositivity and malaria infection but underscored the low incidence of both malaria and HIV within the 17-26 age range and amongst relatively well-educated individuals. In spite of the low prevalence observed, there is still the need for awareness of HIV and Malaria prevention amongst university students. Further studies could be

\footnotetext{
Correspondence: Iheanyi O. Okonko, Department of Microbiology, University of Port Harcourt, PMB 5323, Choba, Nigeria.

E-mail: iheanyi.okonko@uniport.edu.ng
}

Key words: Antibody, Malaria, HIV-1/2, Plasmodium, Prevalence.

Contributions: the authors contributed equally.

Conflict of interest: the authors declare no potential conflict of interest.

Funding: none.

Received for publication: 10 March 2019.

Revision received: 3 July 2019.

Accepted for publication: 3 July 2019.

${ }^{\circ}$ Copyright: the Author(s), 2019

Licensee PAGEPress, Italy

Microbiologia Medica 2019; 34:8160

doi: $10.4081 / \mathrm{mm} .2019 .8160$

This article is distributed under the terms of the Creative Commons Attribution Noncommercial License (by-nc 4.0) which permits any noncommercial use, distribution, and reproduction in any medium, provided the original author(s) and source are credited. undertaken to investigate other relevant epidemiology parameters with regards to HIV and malaria in the tropics.

\section{Introduction}

Malaria is a vector-borne disease caused by species of the protozoan Plasmodium. Plasmodium falciparum (P. falciparum) has been observed to predominate as the causative agent. Over $50 \%$ of the world's population is vulnerable to malaria infection (1).

There are an estimated 300 million malaria cases annually in the tropics with $90 \%$ of these in the sub-Sahara, a region that suffers the greatest burden of human immunodeficiency virus 1 (HIV-1) infection as well (2,3). Malaria has been included in the list of AIDS-related opportunistic infections by the Centre for Disease Prevention and Control since 2009 (4). The high prevalence of both HIV and malaria in the sub-Saharan regions mean that co-infection is relatively common. The two infections complicate each other making difficult an effective treatment (3). While it is not considered the primary cause of mortality, malaria is the third most significant cause of ill-health in people living with HIV/AIDS (5).

The synergistic nature of the HIV-malaria partnership makes co-infection a major health concern. HIV infection impacts on malaria by suppressing the immune response to Plasmodium invasion. Several researchers maintain that malaria increases both in vivo and in vitro replication of the HIV $(1,6,7)$. This means that HIV positive individuals have a more severe manifestation of malaria than would be observed in an HIV negative individual (8). This is likely due to the adverse effect of HIV on CD4 T cells which play a major role in the development and maintenance of anti-malaria immunity $(9,10)$. Both HIV infection and malaria present with fever and anaemia hampering effective identification as the overlap of symptoms can be misleading; hence the need for proper laboratory investigation prior to diagnosis and treatment. A statistically significant link between HIV status and the likelihood of an erroneous diagnosis of malaria have been reported (11).

The burden of HIV infection and malaria on mortality in Africa, Southeast Asia and South America is enormous. The combination of malaria and HIV was responsible for more than three million deaths in 2007 (12,13). Nigeria which has the largest population of any African country has an HIV prevalence of about $4.6 \%$ with 2.95 million of the over 150 million Nigerians being HIV positive $(1,14)$. The presence of the human immunodeficiency virus facilitates the rapid progression of malaria, enhances its severity and increases the possibility of congenital infection (1). Co-infection has been linked to several complications such as respiratory distress and hypoglycaemia and is particularly associated with anaemia in both HIV positive and negative patients (5). Some 
researchers reported that HIV seropositivity in expectant mothers resulted in high-density $P$. falciparum infection and anaemia which is a risk factor for maternal mortality; there is also a 35\% risk of low infant birth weight $(3,15)$. Stimulation and sensitivity analyses showed that malaria accelerates the development of AIDS from HIV infection (3). Cohort studies in Cameroon showed that malaria infection during pregnancy may increase the risk of mother-tochild transmission of HIV (16).

An overlap has been observed in the geographic distribution of both disease conditions: co-infection has been found to expedite the geographic spread of malaria in areas with elevated HIV incidence $(1,2,17)$. This geographical overlap observed between malaria and HIV infections alludes to some correlation between the two and informs the need to investigate incidence of co-infection. Majority of studies on HIV-Malaria co-infection in Africa concentrate on the south and east Africa (1). This study assessed the prevalence of HIV and malaria infections in a cohort of 200 undergraduates from the University of Port Harcourt in southern Nigeria.

\section{Materials and Methods}

\section{Study area}

The study was conducted at the Health Centre of the University of Port Harcourt, Choba, Nigeria. Port Harcourt is the capital city of Rivers State and is located in the forest zone of south Southern Nigeria.

\section{Study design}

Two hundred (200) blood samples were collected from undergraduate students (100 females and 100 males) who were undergoing medical examination at the Health Centre of the University of Port Harcourt. The study covers age range 17-26 years. The minimum sample size was derived using the formula:

$n=\mathrm{PQ}$

$(e / \overline{1.96})^{2}$

where $\mathrm{n}=$ sample size; $\mathrm{P}^{*}=$ working proportion $=7.3 \% ; \mathrm{Q}=100$ $-\mathrm{P}=100-7.3=92.7 \%$; $\mathrm{e}=$ margin of sampling error tolerated $(5 \%)$, at $95 \%$ degree of confidence.

*The working proportion $(\mathrm{P})$ was determined by the relative prevalence of HIV based on other studies in the region. The value calculated was approximated to 200 to make room for further possible errors.

\section{Ethical considerations}

Questionnaires were used to anonymously collect students' demographic variables and other relevant information. All patients indicated their consent to participate in the study by filling out consent forms. All those unwilling to provide informed consent were excluded from the study.

\section{Assay for malaria antigen and HIV antibodies}

Venous blood $(3.0 \mathrm{~mL})$ was collected from participants into EDTA bottle according to Cheesbrough (18) and parallel tests were conducted for each plasma sample to detect both malaria antigen and HIV antibodies in plasma using SD Boline malaria Antigen P.F. test kit and Determine ${ }^{\circledR}$ HIV one step rapid Kit respectively. These immuno-chromatographic kits were used in a stepwise order for the detection of HIV antibody and Malaria antigen in the blood, having $99.4 \%$ sensitivity and $99.6 \%$ specificity and more than $99.9 \%$ sensitivity and $98.6 \%$ specificity respectively. The test and interpretation of the results were done in accordance with the guidelines of the Kits' manufacturers.

\section{Microscopic confirmation of malaria infection}

With blood samples that showed a positive result with the SD Boline malaria Antigen P.F. test kit, microscopic examination was used to confirm the presence of the malaria parasite Plasmodium, as recommended by Kosack et al. (19). The peripheral smear study method was used. Thick and thin blood smears were made on clean glass slides. The thin smears were air dried for 10 minutes then fixed in methanol while the thick smears were dried for 30 minutes without fixing to allow for haemolysis. The smears were stained by immersing in $10 \%$ Giemsa stain for 5 minutes. The smears were then examined under the oil immersion lens of a microscope. This was done solely to confirm the presence of the parasite $(19,20)$.

\section{Statistical analyses}

Statistical Package for the Social Sciences (SPSS)® 21.0 (International Business Machines Corporation (IBM), NY, USA) was used to analyse the data. Statistical significance of data sets was determined at $\mathrm{P} \leq 0.05$.

\section{Results}

None of the participants was on antiretrovirals therapy, none of the participants had recently used any anti-malaria medication and none showed any definite symptoms of malaria or HIV. Three (3) students were positive for malaria of which one (1) was male and the other two (2) female giving prevalence values of $1 \%$ and $2.0 \%$ respectively. Five (5) students were found to be HIV positive: two (2) were female and three (3), male. The overall prevalence for HIV was $2.5 \%$ while the overall prevalence for malaria was $1.5 \%$. The resulting prevalence of HIV positivity based on gender was $2 \%$ and $3 \%$ for females and males respectively. No incident of co-infection was recorded in this study. The findings did not indicate any relationship between HIV seropositivity and malaria infection. Statistical analyses indicated that there were no significant differences between sexes for either malaria or HIV infection. The results are illustrated in Figures 1 and 2.

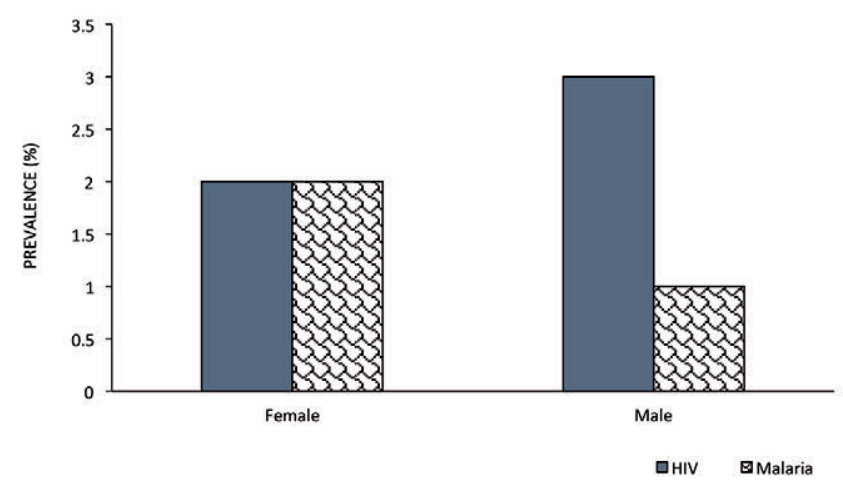

Figure 1. Observed prevalence of malaria and HIV among the students. 


\section{Discussion}

The prevalence values recorded in this study are exceptionally low when assessed against similar studies in Nigeria and across Africa. This is especially true for the observed HIV occurrence. Saracino et al. (5) recorded the prevalence of $66.7 \%$ and $47.2 \%$ for HIV and Malaria respectively; incidence of co-infection in this study was $25.9 \%$ amongst 330 patients attending the clinic in Mozambique (5). With malaria, Fana et al. (21) reported a $41.6 \%$ prevalence in a study of 255 pregnant women in Kebbi state, Nigeria. The prevalence of malaria amongst 1190 adults in Kenya was $28 \%$ (22). A much higher malaria prevalence value of $78.7 \%$ was recorded in a study in Akure, Nigeria, while researchers in southern Ethiopia found the prevalence of malaria in Ethiopia to be $6.1 \%(23,24)$. The overall prevalence of HIV in Rivers state, Nigeria as at 2012 was asserted to be within the range of $4.1-6.0 \%$ (25) which is still higher than found in this study.

The low prevalence of HIV in this study is consistent with several reports in Nigeria and sub-Saharan Africa that have associated HIV incidence with blue-collar workers who often have a minimal formal education. This observation was attributed to their lack of awareness of HIV prevention and control methods and the reliance on nonorthodox forms of protection from sexually transmitted infection (STI). These individuals have further been associated with hesitation to accept their status and attend the associated clinics (26-29). Bluecollar workers often work outside exposing them to malaria infection. This study examined undergraduate students and thus, the observed low prevalence values should be expected. The low prevalence indicates their somewhat more advanced understanding of the prevention and control of HIV as well as appreciation of safe sexual practices.

The low prevalence observed could also be explained by the ages of the participants. This study comprised solely individuals within the usual undergraduate 17-26 age range. The age range has been highlighted in several studies as being less vulnerable to both malaria and HIV infection. The mean age for malaria infection has been found to be about 34 years. Malaria positivity is predominant in age groups 30-34 years; the 25-29 and 35-39 range followed closely $(3,5)$. Singh et al. $(30)$ confirmed the mean age for HIV infection to be 43.3 years with $73.8 \%$ of HIV positivity in the $41-$ 60 age range. The greatest number of HIV positive individuals were found to lie within the 30-49 age range (31). These findings are further buttressed by other similar studies (25,32-34).

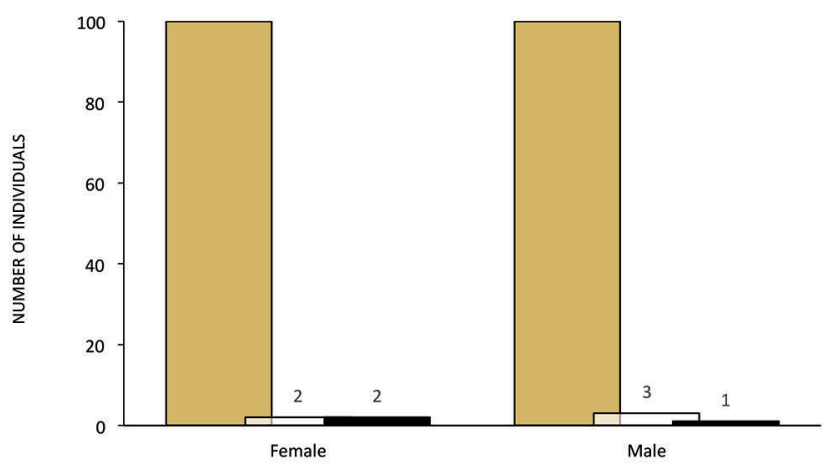

口Number Tested

QNumber Positive for HIV

- Number Positive for Malaria

Figure 2. Distribution of malaria and HIV among students with respect to gender.
The occurrence of HIV-Malaria co-infection is commonly relatively low. A study in Lagos, Nigeria was found to report a prevalence of $2.9 \%$ HIV-malaria co-infection amongst 1080 patients (1). Other studies found that no significant statistical difference existed between the prevalence of malaria in HIV positive and HIV negative individuals $(1,35)$. This corroborates the lack of co-infection in this study. There, however, seems to be a link between susceptibility to malaria and HIV seropositivity as the study found that the diagnosis of malaria was $47.7 \%$ in HIV positive individuals compared to $25.8 \%$ in uninfected people. Other researchers confirm this observation $(15,36)$. The prevalence of malaria infection among HIV-infected persons in Mozambique and South Africa was considerably high $(37,38)$. Likewise observed prevalence in a study in South Eastern Nigeria found co-infection amongst asymptomatic HIV seropositive patients to be $11.8 \%$ (39). Naing et al. (40) recorded an overall co-infection prevalence of $19 \%$ in adults, $12 \%$ in pregnant women, and $9 \%$ in children.

Gender has been highlighted as an important risk factor in the prevalence of both malaria and HIV infection with women being $50 \%$ more likely to contract malaria than men (22). In contrast to this study, some researchers opine that females are more prone to HIV infection. In one study, the prevalence of HIV in males was found to be higher compared to females with values of $67.5 \%$ and $32.5 \%$ respectively (41); another study in contrast reported higher HIV prevalence of $44.83 \%$ in females and $38.3 \%$ in males (42). A publication by the UNAIDS in 2017 confirmed that men generally were more hesitant when it came to HIV testing and accessing ART making them more prone to the disease than women as women will often start the treatment in time and sustain treatment without interruptions. "Globally, less than half of men living with HIV are on treatment, compared to $60 \%$ of women" (43). Furthermore, in developing countries, women are more sexually reserved. They are typically infected secondarily by their partners and are diagnosed following the diagnosis of their partners (30).

With malaria, women tend to dominate in incidence statistics. This higher prevalence in females has been attributed to the fact that females in developing countries are often more involved in domestic activities which keep them in somewhat constant contact with their outdoor environment well into the evenings when mosquitoes are more active (23). Akin to the current findings, females had $25.6 \%$ higher incidence of malaria than males in a study in Akure, Nigeria (23). Other similar studies affirm that females had a higher prevalence of malaria infection compared to their male counterparts but also highlight the fact that, similar to our study, there was no statistically significant difference between the malaria prevalence rate of females and males $(3,5)$. Abdulahi et al. (44) caution that though certain genders could predominate in studies on malaria and co-infection, gender has no significant influence over the incidence of malaria.

The individuals with a positive result for malaria in this study seem to be asymptomatic carriers of the parasite as they do not show any clinical signs of malaria. This is not uncommon in malaria endemic regions. Several studies highlight the importance of these asymptomatic carriers in prevalence studies as it is thought that they serve as reservoirs for the parasite. While none of the patients in this study was on antiretroviral therapy (ART) or antimalarials, the employment of these infection management regimen reduces diagnosis of malaria in HIV positive individuals, especially when used regularly. Co-trimoxazole (CTX) treatment for malaria and antiretroviral therapy (ART) have been found to offer an effective defence against malaria in HIV positive individuals effect from malaria $(8,45,46)$. Saracino et al. (5) found that HIVinfected patients on daily cotrimoxazole treatment were about $20 \%$ less likely to contract malaria. 


\section{Conclusions}

This study found that the prevalence of both malaria and HIV were relatively low amongst undergraduates at the University of Port Harcourt. Females were found to be more prone to malaria infection while their male counterparts demonstrated a greater disposition to HIV infection. HIV status did not seem to influence predisposition to malaria infection as no increase in susceptibility was observed with HIV positive students. It further highlighted the prospect that malaria was not necessarily a health concern with HIV positive undergraduates. A word of caution though - no definite conclusions can be drawn regarding the interaction between HIV and malaria during co-infection because of the low numbers of HIV seropositive individuals recorded in the study. Further studies could be undertaken to investigate other epidemiology parameters and are needed on methods to rapidly estimate needs (incidence) and coverage along with strategies to efficiently expand treatment access.

\section{References}

1. Sanyaolu AO, Fagbenro-Beyioku AF, Oyibo WA, et al. Malaria and HIV co-infection and their effect on haemoglobin levels from three healthcare institutions in Lagos, southwest Nigeria. Afr Health Sci 2013;13:295-300.

2. Cuadros DF, Branscum AJ, Crowley PH. HIV malaria coinfection: effects of malaria on the prevalence of HIV in East sub-Saharan Africa. Int J Epidemiol 2011;40:931-9.

3. Tay SCK, Badu K, Mensah AA, Gbedema SY. The prevalence of malaria among HIV seropositive individuals and the impact of the co- infection on their hemoglobin levels. Ann Clin Microbiol Antimicrob 2015;14:10.

4. Centers for Disease Control and Prevention, CDC. Guidelines for prevention and treatment of opportunistic infections in HIV-infected adults and adolescents. mMWR 2009;58:94-8.

5. Saracino A, Nacarapa EA, da Costa Massinga EA, et al. Prevalence and clinical features of HIV and malaria co-infection in hospitalized adults in Beira, Mozambique. Malaria $\mathrm{J}$ 2012;11:241.

6. Pisell T, Hoffman I, Jere C. Immune activation and induction of HIV-1 replication within CD14 macrophages during acute Plasmodium falciparum malaria coinfection. AIDS 2005;16: 1503-9.

7. Reina L, Potter S. Co-infection of malaria with HIV: an immunological perspective. Immunology 2003;28:589-95.

8. Flateau C, Le Loup G, Pialoux G. Consequences of HIV infection on malaria and therapeutic implications: a systematic review. Lancet Infect Dis 2011;11:541-56.

9. French N, Nakiyingi J, Lugada E, et al. Increasing rates of malarial fever with deteriorating immune status in HIV-1infected Ugandan adults. AIDS 2001;15:899-906.

10. Perrault SD, Hajek J, Zhong K, et al. Human immunodeficiency virus coinfection increases placental parasite density and transplacental malaria transmission in Western Kenya. Am J Trop Med Hyg 2009;80:119-25.

11. Berg A, Patel S, Langeland N, Blomberg B. Falciparum malaria and HIV-1 in hospitalized adults in Maputo, Mozambique: does HIV-infection obscure the malaria diagnosis? Malaria J 2008;7:252.

12. World Health Organisation, WHO. World Malaria Report 2008. WHO Press, Geneva, Switzerland; 2008.
13. World Health Organisation, WHO/UNAIDS/UNICEF. Epidemiological fact sheet on HIV and AIDS. Core data on epidemiology and response Nigeria 2008 update (online); 2008. Available from: http:// apps.who.int/globalatlas/predefined Reports/EFS2008/full/EFS2008_NG.pdf.

14. World Health Organisation, WHO. World Malaria Report 2011. World Global Malaria Programme, WHO Press, Geneva, Switzerland; 2011.

15. Hochman S, Kim K. The Impact of HIV and Malaria Coinfection: What Is Known and Suggested Venues for Further Study. Interdisciplinary Perspectives on Infectious Diseases 2009; Article ID 617954.

16. Alemu A, Shiferaw Y, Addis Z. Effect of malaria on HIV/AIDS transmission and progression. Parasites Vectors 2013;6:18.

17. United Nations AIDS Programme, UNAIDS. World AIDS Day report 2012, Regional fact sheets; 2012. Available from http://www.unaids.org/enresources/campaigns/20121120_glob alreport2012/factsheet/. Accessed: 10th May, 2018.

18. Cheesbrough M. District Laboratory Practice in Tropical Countries, Part 2. 2nd Ed. Cambridge University Press, United Kingdom; 2006.

19. Kosack CS, Naing WT, Piriou E, Shanks L. Routine parallel diagnosis of malaria using microscopy and the malaria rapid diagnostic test SD 05FK60: the experience of Médecins sans Frontières in Myanmar. Malaria J 2013;12:167.

20. Siahaan L. Laboratory diagnostics of malaria. IOP Conference series: Earth and Environmental Sciences 2018;125:012090.

21. Fana SA, Bunza MDA, Anka SA, Usman AU. Prevalence and risk factors associated with malaria infection among pregnant women in a semi-urban community of north-western Nigeria. Infect Dis Poverty 2015;4:24.

22. Jenkins R, Omollo R, Ongecha, M, et al. Prevalence of malaria parasites in adults and its determinants in malaria endemic area of Kisumu county, Kenya. Malaria J 2015;14:263.

23. Obimakinde ET, Simon-Oke IA. The prevalence of malaria infection among patients attending the health centre of the Federal University of Technology, Akure, Nigeria. Int J Trop Dis Health 2017;27:1-7.

24. Debo GW, Kassa DH. Prevalence of malaria and associated factors in Benna Tsemay District of pastoralist community, Southern Ethiopia. Trop Dis Travel Med Vaccines 2016;2:16.

25. National Agency for the Control of AIDS, NACA. Global AIDS Response: Country Progress Report, GARPR, Abuja, Nigeria; 2012.

26. Mohanty KC, Sundrani RM, Nair S. HIV infection in patients with respiratory disease. Indian J Tuberc 1993;40:5.

27. Rajasekaran R, Lima A, Kamakshi S, et al. Trend of HIV infection in patients with tuberculosis in rural south India. Indian J Tuberc 2000;47:223.

28. Bhattacharya MK, Naik TN, Ghosh M, et al. Pulmonary tuberculosis among HIV seropositives attending a counseling center in Kolkata. Indian J Public Health 2011;55:329-31.

29. Okonko IO, Odu NN, Osadebe AU, Anyanwu A. HIV and Tuberculosis Co-Infection in a Highly HIV-Infected Population of Rivers State, Nigeria. J Immunol Immunochem 2018;39:636-46.

30. Singh A, Singh MT, Deepti SS. Socio-demographic and clinical profile of HIV/AIDS patients attending the ART centre of Amritsar, Punjab. Int J Commun Med Public Health 2018;5: 2059-65.

31. Sharma A, Chauhan N. Seroprevalence of HIV among General Population Attending at a Tertiary Care Hospital in Udaipur, Rajasthan, India. Int J Curr Microbiol App Sci 2018;7:1774-9.

32. Awoleye OJ, Thron C. Determinants of Human 
Immunodeficiency Viirus (HIV) Infection in Nigeria: A synthesis of the literature. J AIDS HIV Res 2015;7:117-29.

33. Mishra M, Agrawal S, Qazi MS, et al Department of Microbiology Government Medical College, Maharashtra: Declining HIV seropositivity: Ten years' experience from a tertiary care hospital in central India. Indian $\mathrm{J}$ Med Res 2016;2016:518-20.

34. Sherwal BL, Gupta P, Nayak R, et al. Prevalence of HIV in a Tertiary Care Centre in Delhi: A Five-Year ICTC Based Study. World J AIDS 2015;5:1-9.

35. Hochman S, Kim K. The Impact of HIV Coinfection on Cerebral Malaria Pathogenesis. J Neuroparasitol 2012;3:235547.

36. Idemyor V. Human immunodeficiency virus (HIV) and malaria interaction in sub-Saharan Africa: the collision of two Titans. HIV Clin Trials 2007;8:246-53.

37. Guyatt HL, Snow RW. The epidemiology and burden of Plasmodium falciparum-related anemia among pregnant women in sub-Saharan Africa. Am J Trop Med Hyg 2001;64:36-44.

38. Mocroft A, Kirk O, Barton SE, et al. Anaemia is an independent predictive marker for clinical prognosis in HIV-infected patients from across Europe. EuroSIDA study group. AIDS 1999;13:943-50.

39. Fo TK, Parise ME, Verhoeff FH, et al. The Burden of Co-infection with human immunodeficiency virus type 1 and malaria in pregnant women in Sub-Saharan Africa. Am J Trop Med Hyg 2004;71:41-54.

40. Naing C, Sandhu NK, Wai VN. The Effect of Malaria and HIV Co-Infection on Anemia, a Meta-Analysis. Medicine 2006;95: $1-10$.

41. Sabhapandit D, Hazarika P, Phukan AC, et al. A Study on HIVTB Coinfection among Patients Attending a Tertiary Care Centre in North East India. Int J Health Sci Res 2017;7:94-101.

42. Pennap G, Makpa S, Ogbu S. Sero-prevalence of HIV infection among tuberculosis patients in a rural tuberculosis referral clinic in northern Nigeria. Pan Afr Med J 2010;5:22.

43. Oleribe OO, Aliyu S, Taylor-Robinson SD. Is the prevalence of HIV wrongly estimated in Nigeria? Some insights from a 2017 World AIDS day experience from a Nigerian NonGovernmental Organisation. Pan Afr Med J 2018;29:119.

44. Abdulahi K, Abubakar U, Adamu T, et al. Malaria in Sokoto, North Western Nigeria. Afr J Biotechnol 2009;8:7101-5.

45. Mermin J, Ekwaru JP, Liechty CA, et al. Effect of co-trimoxazole prophylaxis, antiretroviral therapy, and insecticide-treated bednets on the frequency of malaria in HIV-1-infected adults in Uganda: a prospective cohort study. Lancet 2006;367:1256-61.

46. Thera MA, Sehdev PS, Coulibaly D, et al. Impact of trimethoprim-sulfamethoxazole prophylaxis on falciparum malaria infection and disease. J Infect Dis 2005;192:823-9. 